\title{
Molybdenum Oxide, Oxycarbide and Carbide: Controlling the Dynamic Composition, Size and Catalytic Activity of Zeolite-Supported Nanostructures
}

\author{
Yiteng Zheng ${ }^{1}$, Yadan Tang ${ }^{2}$, James R. Gallagher ${ }^{3}$, Jie Gao ${ }^{1}$, \\ Jeffrey T. Miller ${ }^{3,4 *}$, Israel E. Wachs ${ }^{2 *}$, Simon G. Podkolzin ${ }^{1 *}$
}

\section{Supporting Information}

${ }^{1}$ Department of Chemical Engineering and Materials Science, Stevens Institute of Technology, Hoboken, New Jersey 07030 United States.

${ }^{2}$ Operando Molecular Spectroscopy \& Catalysis Laboratory, Department of Chemical and Biomolecular Engineering, Lehigh University, Bethlehem, Pennsylvania 18015 United States.

${ }^{3}$ Chemical Science and Engineering Division, Argonne National Laboratory, Argonne, Illinois 60439 United States.

${ }^{4}$ Davidson School of Chemical Engineering, Purdue University, West Lafayette, Indiana 47907 United States.

*Corresponding authors: S.G.P.: Simon.Podkolzin@Stevens.edu, I.E.W: iew0@lehigh.edu, J.T.M.: jeffrey-t-miller@purdue.edu 


\section{Supporting Information}

The Mo oxycarbide structures in Figure 6 are shown with atom numbering labels in Figure $\mathrm{S} 1$ and their geometries are provided in Tables S1-S5. Bond distances and energies for the transition state structures in Figures 7-10 are provided in Tables S6-S9.

Table S1. Geometry of the oxycarbide structure in Figures 6a and S1a.

\begin{tabular}{|l|c|l|c|}
\hline \multicolumn{2}{|c|}{ Bond distance, $\mathrm{nm}$} & \multicolumn{2}{c|}{ Bond angle, degree } \\
\hline $\mathrm{Mo}(1)-\mathrm{Mo}(2)$ & 0.29 & $\mathrm{C}(1)-\mathrm{Mo}(1)-\mathrm{C}(2)$ & 91 \\
\hline $\mathrm{Mo}(1)-\mathrm{Mo}(3)$ & 0.24 & $\mathrm{Mo}(3)-\mathrm{Mo}(1)-\mathrm{Mo}(4)$ & 51 \\
\hline $\mathrm{Mo}(1)-\mathrm{Mo}(4)$ & 0.29 & $\mathrm{C}(1)-\mathrm{Mo}(2)-\mathrm{C}(2)$ & 85 \\
\hline $\mathrm{Mo}(2)-\mathrm{Mo}(3)$ & 0.30 & $\mathrm{Mo}(3)-\mathrm{Mo}(2)-\mathrm{Mo}(4)$ & 49 \\
\hline $\mathrm{Mo}(2)-\mathrm{Mo}(4)$ & 0.25 & $\mathrm{Mo}(1)-\mathrm{Mo}(2)-\mathrm{O}$ & 151 \\
\hline $\mathrm{Mo}(3)-\mathrm{Mo}(4)$ & 0.23 & $\mathrm{Mo}(3)-\mathrm{Mo}(2)-\mathrm{O}$ & 159 \\
\hline $\mathrm{Mo}(1)-\mathrm{C}(1)$ & 0.19 & $\mathrm{Mo}(1)-\mathrm{Mo}(3)-\mathrm{Mo}(2)$ & 64 \\
\hline $\mathrm{Mo}(1)-\mathrm{C}(2)$ & 0.19 & $\mathrm{C}(1)-\mathrm{Mo}(3)-\mathrm{Mo}(4)$ & 93 \\
\hline $\mathrm{Mo}(2)-\mathrm{C}(1)$ & 0.20 & $\mathrm{Mo}(1)-\mathrm{Mo}(4)-\mathrm{Mo}(2)$ & 64 \\
\hline $\mathrm{Mo}(2)-\mathrm{C}(2)$ & 0.21 & $\mathrm{C}(2)-\mathrm{Mo}(4)-\mathrm{Mo}(3)$ & 92 \\
\hline $\mathrm{Mo}(3)-\mathrm{C}(1)$ & 0.22 & & \\
\hline $\mathrm{Mo}(4)-\mathrm{C}(2)$ & 0.21 & & \\
\hline $\mathrm{Mo}(2)-\mathrm{O}$ & 0.18 & & \\
\hline
\end{tabular}

Table S2. Geometry of the oxycarbide structure in Figures $6 b$ and S1b.

\begin{tabular}{|l|c|c|c|}
\hline \multicolumn{2}{|c|}{ Bond distance, $\mathrm{nm}$} & \multicolumn{2}{c|}{ Bond angle, degree } \\
\hline $\mathrm{Mo}(1)-\mathrm{Mo}(2)$ & 0.28 & $\mathrm{C}(1)-\mathrm{Mo}(1)-\mathrm{C}(2)$ & 88 \\
\hline $\mathrm{Mo}(1)-\mathrm{Mo}(3)$ & 0.24 & $\mathrm{Mo}(3)-\mathrm{Mo}(1)-\mathrm{Mo}(4)$ & 50 \\
\hline $\mathrm{Mo}(1)-\mathrm{Mo}(4)$ & 0.30 & $\mathrm{C}(1)-\mathrm{Mo}(2)-\mathrm{C}(2)$ & 88 \\
\hline $\mathrm{Mo}(2)-\mathrm{Mo}(3)$ & 0.29 & $\mathrm{Mo}(3)-\mathrm{Mo}(2)-\mathrm{Mo}(4)$ & 51 \\
\hline $\mathrm{Mo}(2)-\mathrm{Mo}(4)$ & 0.25 & $\mathrm{Mo}(1)-\mathrm{Mo}(3)-\mathrm{Mo}(2)$ & 63 \\
\hline $\mathrm{Mo}(3)-\mathrm{Mo}(4)$ & 0.24 & $\mathrm{C}(1)-\mathrm{Mo}(3)-\mathrm{Mo}(4)$ & 95 \\
\hline $\mathrm{Mo}(1)-\mathrm{C}(1)$ & 0.20 & $\mathrm{Mo}(1)-\mathrm{Mo}(4)-\mathrm{Mo}(2)$ & 60 \\
\hline $\mathrm{Mo}(1)-\mathrm{C}(2)$ & 0.19 & $\mathrm{C}(2)-\mathrm{Mo}(4)-\mathrm{Mo}(3)$ & 93 \\
\hline $\mathrm{Mo}(2)-\mathrm{C}(1)$ & 0.19 & $\mathrm{Mo}(2)-\mathrm{Mo}(4)-\mathrm{O}$ & 129 \\
\hline $\mathrm{Mo}(2)-\mathrm{C}(2)$ & 0.20 & $\mathrm{Mo}(3)-\mathrm{Mo}(4)-\mathrm{O}$ & 120 \\
\hline $\mathrm{Mo}(3)-\mathrm{C}(1)$ & 0.22 & & \\
\hline $\mathrm{Mo}(4)-\mathrm{C}(2)$ & 0.24 & & \\
\hline $\mathrm{Mo}(4)-\mathrm{O}$ & 0.18 & & \\
\hline
\end{tabular}


Table S3. Geometry of the oxycarbide structure in Figures 6c and S1c.

\begin{tabular}{|l|c|l|c|}
\hline \multicolumn{2}{|c|}{ Bond distance, $\mathrm{nm}$} & \multicolumn{2}{c|}{ Bond angle, degree } \\
\hline $\mathrm{Mo}(1)-\mathrm{Mo}(2)$ & 0.27 & $\mathrm{C}(1)-\mathrm{Mo}(1)-\mathrm{C}(2)$ & 87 \\
\hline $\mathrm{Mo}(1)-\mathrm{Mo}(3)$ & 0.25 & $\mathrm{Mo}(3)-\mathrm{Mo}(1)-\mathrm{Mo}(4)$ & 50 \\
\hline $\mathrm{Mo}(1)-\mathrm{Mo}(4)$ & 0.28 & $\mathrm{Mo}(2)-\mathrm{Mo}(1)-\mathrm{O}$ & 120 \\
\hline $\mathrm{Mo}(2)-\mathrm{Mo}(3)$ & 0.30 & $\mathrm{Mo}(3)-\mathrm{Mo}(1)-\mathrm{O}$ & 50 \\
\hline $\mathrm{Mo}(2)-\mathrm{Mo}(4)$ & 0.24 & $\mathrm{Mo}(4)-\mathrm{Mo}(1)-\mathrm{O}$ & 81 \\
\hline $\mathrm{Mo}(3)-\mathrm{Mo}(4)$ & 0.23 & $\mathrm{C}(1)-\mathrm{Mo}(2)-\mathrm{C}(2)$ & 90 \\
\hline $\mathrm{Mo}(1)-\mathrm{C}(1)$ & 0.19 & $\mathrm{Mo}(3)-\mathrm{Mo}(2)-\mathrm{Mo}(4)$ & 47 \\
\hline $\mathrm{Mo}(1)-\mathrm{C}(2)$ & 0.20 & $\mathrm{Mo}(1)-\mathrm{Mo}(3)-\mathrm{Mo}(2)$ & 58 \\
\hline $\mathrm{Mo}(2)-\mathrm{C}(1)$ & 0.20 & $\mathrm{C}(1)-\mathrm{Mo}(3)-\mathrm{Mo}(4)$ & 89 \\
\hline $\mathrm{Mo}(2)-\mathrm{C}(2)$ & 0.19 & $\mathrm{Mo}(2)-\mathrm{Mo}(3)-\mathrm{O}$ & 109 \\
\hline $\mathrm{Mo}(3)-\mathrm{C}(1)$ & 0.23 & $\mathrm{Mo}(1)-\mathrm{Mo}(4)-\mathrm{Mo}(2)$ & 62 \\
\hline $\mathrm{Mo}(4)-\mathrm{C}(2)$ & 0.26 & $\mathrm{C}(2)-\mathrm{Mo}(4)-\mathrm{Mo}(3)$ & 97 \\
\hline $\mathrm{Mo}(1)-\mathrm{O}$ & 0.20 & $\mathrm{Mo}(1)-\mathrm{O}-\mathrm{Mo}(3)$ & 78 \\
\hline $\mathrm{Mo}(3)-\mathrm{O}$ & 0.20 & & \\
\hline
\end{tabular}

Table S4. Geometry of the oxycarbide structure in Figures 6d and S1d.

\begin{tabular}{|l|c|l|c|}
\hline \multicolumn{2}{|c|}{ Bond distance, $\mathrm{nm}$} & \multicolumn{2}{c|}{ Bond angle, degree } \\
\hline $\mathrm{Mo}(1)-\mathrm{Mo}(2)$ & 0.28 & $\mathrm{C}(1)-\mathrm{Mo}(1)-\mathrm{C}(2)$ & 86 \\
\hline $\mathrm{Mo}(1)-\mathrm{Mo}(3)$ & 0.28 & $\mathrm{Mo}(2)-\mathrm{Mo}(1)-\mathrm{Mo}(3)$ & 57 \\
\hline $\mathrm{Mo}(1)-\mathrm{Mo}(4)$ & 0.24 & $\mathrm{Mo}(3)-\mathrm{Mo}(1)-\mathrm{Mo}(4)$ & 56 \\
\hline $\mathrm{Mo}(2)-\mathrm{Mo}(3)$ & 0.26 & $\mathrm{C}(1)-\mathrm{Mo}(1)-\mathrm{Mo}(3)$ & 43 \\
\hline $\mathrm{Mo}(2)-\mathrm{Mo}(4)$ & 0.24 & $\mathrm{C}(2)-\mathrm{Mo}(1)-\mathrm{Mo}(2)$ & 43 \\
\hline $\mathrm{Mo}(3)-\mathrm{Mo}(4)$ & 0.25 & $\mathrm{C}(1)-\mathrm{Mo}(2)-\mathrm{Mo}(3)$ & 90 \\
\hline $\mathrm{Mo}(1)-\mathrm{C}(1)$ & 0.19 & $\mathrm{Mo}(3)-\mathrm{Mo}(2)-\mathrm{Mo}(4)$ & 58 \\
\hline $\mathrm{Mo}(1)-\mathrm{C}(2)$ & 0.19 & $\mathrm{Mo}(1)-\mathrm{Mo}(2)-\mathrm{O}$ & 105 \\
\hline $\mathrm{Mo}(2)-\mathrm{C}(2)$ & 0.19 & $\mathrm{Mo}(3)-\mathrm{Mo}(2)-\mathrm{O}$ & 49 \\
\hline $\mathrm{Mo}(3)-\mathrm{C}(1)$ & 0.19 & $\mathrm{Mo}(1)-\mathrm{Mo}(4)-\mathrm{Mo}(3)$ & 69 \\
\hline $\mathrm{Mo}(2)-\mathrm{O}$ & 0.20 & $\mathrm{Mo}(2)-\mathrm{Mo}(4)-\mathrm{Mo}(3)$ & 65 \\
\hline $\mathrm{Mo}(3)-\mathrm{O}$ & 0.20 & & \\
\hline
\end{tabular}


Table S5. Geometry of the oxycarbide structure in Figures 6e and S1e.

\begin{tabular}{|l|l|l|c|}
\hline \multicolumn{2}{|c|}{ Bond distance, $\mathrm{nm}$} & \multicolumn{2}{c|}{ Bond angle, degree } \\
\hline $\mathrm{Mo}(1)-\mathrm{Mo}(2)$ & 0.27 & $\mathrm{C}(1)-\mathrm{Mo}(1)-\mathrm{C}(2)$ & 97 \\
\hline $\mathrm{Mo}(1)-\mathrm{Mo}(3)$ & 0.24 & $\mathrm{Mo}(3)-\mathrm{Mo}(1)-\mathrm{Mo}(4)$ & 52 \\
\hline $\mathrm{Mo}(1)-\mathrm{Mo}(4)$ & 0.28 & $\mathrm{C}(1)-\mathrm{Mo}(2)-\mathrm{C}(2)$ & 96 \\
\hline $\mathrm{Mo}(2)-\mathrm{Mo}(3)$ & 0.29 & $\mathrm{Mo}(3)-\mathrm{Mo}(2)-\mathrm{Mo}(4)$ & 52 \\
\hline $\mathrm{Mo}(2)-\mathrm{Mo}(4)$ & 0.23 & $\mathrm{Mo}(1)-\mathrm{Mo}(3)-\mathrm{Mo}(2)$ & 61 \\
\hline $\mathrm{Mo}(3)-\mathrm{Mo}(4)$ & 0.23 & $\mathrm{C}(1)-\mathrm{Mo}(3)-\mathrm{Mo}(4)$ & 94 \\
\hline $\mathrm{Mo}(1)-\mathrm{C}(1)$ & 0.21 & $\mathrm{Mo}(1)-\mathrm{Mo}(4)-\mathrm{Mo}(2)$ & 62 \\
\hline $\mathrm{Mo}(1)-\mathrm{C}(2)$ & 0.21 & $\mathrm{C}(2)-\mathrm{Mo}(4)-\mathrm{Mo}(3)$ & 96 \\
\hline $\mathrm{Mo}(2)-\mathrm{C}(1)$ & 0.20 & $\mathrm{Mo}(1)-\mathrm{C}(2)-\mathrm{O}$ & 142 \\
\hline $\mathrm{Mo}(2)-\mathrm{C}(2)$ & 0.22 & $\mathrm{Mo}(2)-\mathrm{C}(2)-\mathrm{O}$ & 137 \\
\hline $\mathrm{Mo}(3)-\mathrm{C}(1)$ & 0.21 & $\mathrm{Mo}(4)-\mathrm{C}(2)-\mathrm{O}$ & 129 \\
\hline $\mathrm{Mo}(4)-\mathrm{C}(2)$ & 0.25 & & \\
\hline $\mathrm{C}(2)-\mathrm{O}$ & 0.12 & & \\
\hline
\end{tabular}


Table S6. Bond distances and energies for the structures in $\mathrm{CH}_{4}$ activation over the Mo-C site in $\mathrm{Mo}_{4} \mathrm{C}_{2} / \mathrm{ZSM}-5$ shown in Figure 7.

\begin{tabular}{|l|c|c|c|c|}
\hline & $\begin{array}{c}\mathrm{CH}_{3}-\mathrm{H}, \\
\mathrm{nm}\end{array}$ & $\begin{array}{c}\mathrm{C}-\mathrm{H}, \\
\mathrm{nm}\end{array}$ & $\begin{array}{c}\mathrm{Mo}^{-} \mathrm{CH}_{3}, \\
\mathrm{~nm}\end{array}$ & $\begin{array}{c}\Delta \mathrm{E}, \\
\mathrm{kJ} / \mathrm{mol}\end{array}$ \\
\hline (a) $\mathrm{CH}_{4}+\mathrm{Mo}_{4} \mathrm{C}_{2} / \mathrm{ZSM}-5$ & 0.11 & 0.38 & 0.35 & 0 \\
\hline (b) Intermediate & 0.12 & 0.29 & 0.25 & 20 \\
\hline (c) Transition state & 0.16 & 0.15 & 0.24 & 112 \\
\hline (d) Intermediate & 0.17 & 0.12 & 0.22 & 108 \\
\hline (e) $\mathrm{CH}_{3}+\mathrm{H} / \mathrm{Mo}_{4} \mathrm{C}_{2} / \mathrm{ZSM}-5$ & 0.47 & 0.11 & 0.22 & -46 \\
\hline
\end{tabular}

Table S7. Bond distances and energies for structures in $\mathrm{CH}_{4}$ activation over the Mo-C site in $\mathrm{Mo}_{4} \mathrm{C}_{2} \mathrm{O} / \mathrm{ZSM}-5$ shown in Figure 8.

\begin{tabular}{|l|c|c|c|c|}
\hline & $\begin{array}{c}\mathrm{CH}_{3}-\mathrm{H}, \\
\mathrm{nm}\end{array}$ & $\begin{array}{c}\mathrm{C}-\mathrm{H}, \\
\mathrm{nm}\end{array}$ & $\begin{array}{c}\mathrm{Mo}-\mathrm{CH}_{3}, \\
\mathrm{~nm}\end{array}$ & $\begin{array}{c}\Delta \mathrm{E}, \\
\mathrm{kJ} / \mathrm{mol}\end{array}$ \\
\hline (a) $\mathrm{CH}_{4}+\mathrm{Mo}_{4} \mathrm{C}_{2} \mathrm{O} / \mathrm{ZSM}-5$ & 0.11 & 0.41 & 0.41 & 0 \\
\hline (b) Intermediate & 0.12 & 0.24 & 0.25 & 16 \\
\hline (c) Transition state & 0.16 & 0.15 & 0.23 & 111 \\
\hline (d) Intermediate & 0.17 & 0.13 & 0.22 & 106 \\
\hline (e) $\mathrm{CH}_{3}+\mathrm{H} / \mathrm{Mo}_{4} \mathrm{C}_{2} \mathrm{O} / \mathrm{ZSM}-5$ & 0.44 & 0.11 & 0.21 & -70 \\
\hline
\end{tabular}

Table S8. Bond distances and energies for structures in $\mathrm{CH}_{4}$ activation over the Mo-O site in $\mathrm{Mo}_{4} \mathrm{C}_{2} \mathrm{O} / \mathrm{ZSM}-5$ shown in Figure 9.

\begin{tabular}{|l|c|c|c|c|}
\hline & $\begin{array}{c}\mathrm{CH}_{3}-\mathrm{H}, \\
\mathrm{nm}\end{array}$ & $\begin{array}{c}\mathrm{O}-\mathrm{H}, \\
\mathrm{nm}\end{array}$ & $\begin{array}{c}\mathrm{Mo}^{-\mathrm{CH}_{3},} \\
\mathrm{~nm}\end{array}$ & $\begin{array}{c}\Delta \mathrm{E}, \\
\mathrm{kJ} / \mathrm{mol}\end{array}$ \\
\hline (a) $\mathrm{CH}_{4}+\mathrm{Mo}_{4} \mathrm{C}_{2} \mathrm{O} / \mathrm{ZSM}-5$ & 0.11 & 0.41 & 0.41 & 0 \\
\hline (b) Intermediate & 0.12 & 0.29 & 0.25 & 16 \\
\hline (c) Transition state & 0.14 & 0.12 & 0.23 & 136 \\
\hline (d) Intermediate & 0.17 & 0.10 & 0.22 & 103 \\
\hline (e) $\mathrm{CH}_{3}+\mathrm{H} / \mathrm{Mo}_{4} \mathrm{C}_{2} \mathrm{O} /$ ZSM-5 & 0.45 & 0.10 & 0.22 & -29 \\
\hline
\end{tabular}


Table S9. Bond distances and energies for structures in $\mathrm{CH}_{4}$ activation over the Mo-C site in $\mathrm{Mo}_{4} \mathrm{C}_{4} / \mathrm{ZSM}-5$ shown in Figure 10.

\begin{tabular}{|l|c|c|c|c|}
\hline & $\begin{array}{c}\mathrm{CH}_{3}-\mathrm{H}, \\
\mathrm{nm}\end{array}$ & $\begin{array}{c}\mathrm{C}-\mathrm{H}, \\
\mathrm{nm}\end{array}$ & $\begin{array}{c}\mathrm{Mo}-\mathrm{CH}_{3}, \\
\mathrm{~nm}\end{array}$ & $\begin{array}{c}\Delta \mathrm{E}, \\
\mathrm{kJ} / \mathrm{mol}\end{array}$ \\
\hline (a) $\mathrm{CH}_{4}+\mathrm{Mo}_{4} \mathrm{C}_{4} / \mathrm{ZSM}-5$ & 0.11 & 0.32 & 0.40 & 0 \\
\hline (b) Intermediate & 0.12 & 0.25 & 0.25 & 20 \\
\hline (c) Transition state & 0.15 & 0.14 & 0.23 & 113 \\
\hline (d) Intermediate & 0.17 & 0.11 & 0.22 & 51 \\
\hline (e) $\mathrm{CH}_{3}+\mathrm{H} / \mathrm{Mo}_{4} \mathrm{C}_{4} / \mathrm{ZSM}-5$ & 0.41 & 0.11 & 0.21 & -118 \\
\hline
\end{tabular}
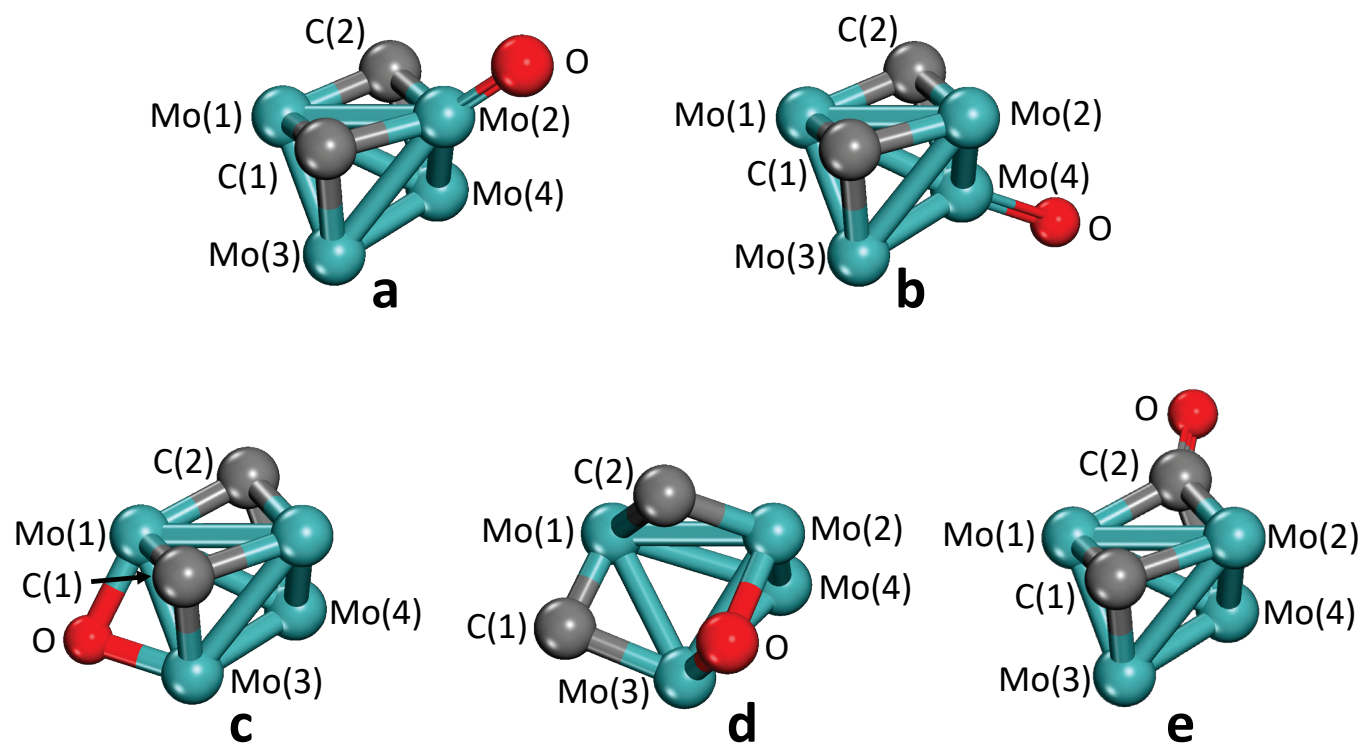

Figure S1. $\mathrm{Mo}_{4} \mathrm{C}_{2} \mathrm{O}$ oxycarbide structures identified with the genetic algorithm with atom numbering labels for the bond distances and angles in Tables S1-S5. 